\title{
Pressure-Induced Polymorphic Transformations of Ethylenediamine Bisborane
}

\author{
Rongfeng Guan, Pan Wang, Yang Song, and Viktor N. Staroverov \\ Department of Chemistry, The University of Western Ontario, London, Ontario N6A 5B7, Canada
}

\section{Contents}

Table S1. Assignment of vibrational frequencies of EDAB by referencing those of amino borane observed experimentally at ambient pressure.

Table S2. Pressure dependence of selected observed IR modes of EDAB.

Table S3. Pressure dependence of selected observed Raman modes of EDAB.

Figure S1. Pressure dependence of the observed vibrational Raman modes of EDAB.

Table S4. Calculated vdW-DF lattice parameters of the 14 candidate structures for phase II at $1.72 \mathrm{GPa}$.

Table S5. Calculated vdW-DF lattice parameters of the 17 candidate structures for phase III at 11.44 GPa.

Table S6. Calculated vdW-DF enthalpies of the final 7 candidate EDAB structures relative to the enthalpy of the reference Pbca structure at the corresponding pressure.

Figure S2. Calculated PBE enthalpies of the final 7 candidate EDAB structures relative to the enthalpy of the reference Pbca structure at the corresponding pressure.

Figure S3. Calculated (vdW-DF) $\mathrm{N}-\mathrm{H} \cdots \mathrm{H}-\mathrm{B}$ dihydrogen bond lengths in EDAB vs. pressure.

Table S7. Crystal structures, cell parameters, and Wyckoff positions for the unit cells of the 7 candidate structures of EDAB optimized at ambient pressure using the vdW-DF functional. 
Table S1. Assignment of vibrational frequencies $\left(\mathrm{cm}^{-1}\right)$ of EDAB by referencing those of amino borane observed experimentally at ambient pressure.

\begin{tabular}{|c|c|c|c|c|}
\hline \multirow[t]{2}{*}{ Assignment } & \multicolumn{3}{|c|}{ EDAB } & \multirow{2}{*}{$\frac{\mathrm{NH}_{3} \cdot \mathrm{BH}_{3}{ }^{[23]}}{\operatorname{Raman}\left(\mathrm{cm}^{-1}\right)}$} \\
\hline & $\operatorname{IR}\left(\mathrm{cm}^{-1}\right)$ & Raman $\left(\mathrm{cm}^{-1}\right)$ & $\operatorname{IR}\left(\mathrm{cm}^{-1}\right)$ & \\
\hline \multirow[t]{4}{*}{ Lattice modes } & & 147 & & 208 \\
\hline & & 198 & & 345 \\
\hline & & 267 & & \\
\hline & & 380 & & \\
\hline B-N stretch & 707 & 697 & 799 & 800 \\
\hline C-N stretch & 1046 & 1053 & & \\
\hline C-C stretch & & 1084 & & \\
\hline \multirow[t]{3}{*}{ B-H bend } & 1166 & 1167 & 1186 & 1189 \\
\hline & 1173 & 1179 & & \\
\hline & 1191 & 1197 & & \\
\hline $\mathrm{C}-\mathrm{H}$ bend & 1262 & 1288 & & \\
\hline $\mathrm{C}-\mathrm{H}$ twist & & 1306 & & \\
\hline \multirow[t]{2}{*}{$\mathrm{N}-\mathrm{H}$ bend } & 1361 & & 1343 & 1357 \\
\hline & 1368 & 1355 & & \\
\hline \multirow[t]{2}{*}{ C-H deformation } & & 1443 & & \\
\hline & 1400 & 1448 & & \\
\hline $\mathrm{N}-\mathrm{H}$ deformation & $\begin{array}{l}1578 \\
1585\end{array}$ & 1588 & & \\
\hline \multirow[t]{4}{*}{ B-H stretch } & 2273 & 2270 & & \\
\hline & 2294 & 2327 & & \\
\hline & 2342 & 2347 & 2340 & 2279 \\
\hline & 2396 & 2385 & 2415 & 2318 \\
\hline C-H sym. stretch & 2895 & 2886 & & \\
\hline overtone & 2939 & 2918 & & \\
\hline \multirow[t]{2}{*}{ C-H asym. stretch } & 2965 & 2955 & & \\
\hline & 2992 & 2970 & & \\
\hline N-H sym. str. & 3143 & 3142 & 3337 & 3250 \\
\hline \multirow[t]{2}{*}{$\mathrm{N}-\mathrm{H}$ asym. str. } & 3224 & 3221 & 3386 & 3316 \\
\hline & 3265 & 3252 & & \\
\hline
\end{tabular}


Table S2. Pressure dependence of selected observed IR modes of EDAB.

\begin{tabular}{|c|c|c|c|c|}
\hline \multirow[b]{2}{*}{ Assignment } & \multirow{2}{*}{$\begin{array}{l}\text { Frequency } \\
\qquad\left(\mathrm{cm}^{-1}\right)\end{array}$} & \multicolumn{3}{|c|}{$d v / d P\left(\mathrm{~cm}^{-1} \mathrm{GPa}^{-1}\right)$} \\
\hline & & $\begin{array}{c}\text { Phase I } \\
(<0.96 \mathrm{GPa})\end{array}$ & $\begin{array}{c}\text { Phase II } \\
(1.0-7.0 \mathrm{GPa})\end{array}$ & $\begin{array}{r}\text { Phase III } \\
(>7.0 \mathrm{GPa})\end{array}$ \\
\hline B-N str. & 707 & 8.8 & 4.7 & 2.6 \\
\hline B-N str. & 759 & & 8.6 & \\
\hline C-N str. & 1046 & 2.8 & 5.2 & 3.5 \\
\hline \multirow{3}{*}{ B-H bend } & 1173 & 4.4 & 3.9 & \\
\hline & 1191 & 6.4 & 6.9 & \\
\hline & 1262 & -2.6 & 1.3 & -0.1 \\
\hline $\mathrm{C}-\mathrm{H}$ bend & 1361 & -1.4 & 2.0 & 1.4 \\
\hline \multirow{2}{*}{$\mathrm{N}-\mathrm{H}$ bend } & 1562 & & -3.4 & \\
\hline & 1578 & -2.0 & -1.2 & -0.02 \\
\hline \multirow{2}{*}{$\mathrm{N}-\mathrm{H}$ deformation } & 1600 & & & 0.2 \\
\hline & 1610 & & & -0.4 \\
\hline \multirow{2}{*}{ B-H str. } & 2273 & 8.4 & 6.2 & 1.0 \\
\hline & 2342 & 7.7 & 11.9 & 7.5 \\
\hline \multirow{2}{*}{ N-H asym. str. } & 3224 & -2.8 & -4.3 & \\
\hline & 3265 & -12.0 & -2.8 & -0.3 \\
\hline
\end{tabular}

Table S3. Pressure dependence of selected observed Raman modes of EDAB.

\begin{tabular}{|c|c|c|c|c|}
\hline \multirow[b]{2}{*}{ Assignment } & \multirow{2}{*}{$\begin{array}{c}\text { Frequency } \\
\quad\left(\mathrm{cm}^{-1}\right)\end{array}$} & \multicolumn{3}{|c|}{$d v / d P\left(\mathrm{~cm}^{-1} \mathrm{GPa}^{-1}\right)$} \\
\hline & & $\begin{array}{c}\text { Phase I } \\
(<1.0 \mathrm{GPa})\end{array}$ & $\begin{array}{c}\text { Phase II } \\
(1.0-7.0 \mathrm{GPa})\end{array}$ & $\begin{array}{l}\text { Phase III } \\
(>7.0 \mathrm{GPa})\end{array}$ \\
\hline \multirow{2}{*}{ Lattice modes } & 264 & 12.2 & 12.1 & 6.0 \\
\hline & 381 & 4.3 & 5.1 & 0.8 \\
\hline \multirow[t]{3}{*}{ B-N str. } & 697 & 5.4 & 5.3 & 1.0 \\
\hline & 802 & & 10.2 & 4.5 \\
\hline & 890 & & 4.4 & 2.5 \\
\hline C-H deformation & 1464 & & 2.1 & 2.0 \\
\hline $\mathrm{N}-\mathrm{H}$ deformation & 1570 & & -1.4 & 0.5 \\
\hline \multirow{3}{*}{ B-H str. } & 2270 & 11.1 & 8.0 & 4.2 \\
\hline & 2342 & & 10.8 & 6.1 \\
\hline & 2347 & 14.9 & & \\
\hline \multirow{5}{*}{$\mathrm{N}-\mathrm{H}$ str. } & 3116 & & -5.9 & \\
\hline & 3142 & -10.8 & & \\
\hline & 3204 & & -4.0 & -0.3 \\
\hline & 3221 & -5.4 & & \\
\hline & 3252 & -3.5 & -4.1 & -0.6 \\
\hline
\end{tabular}



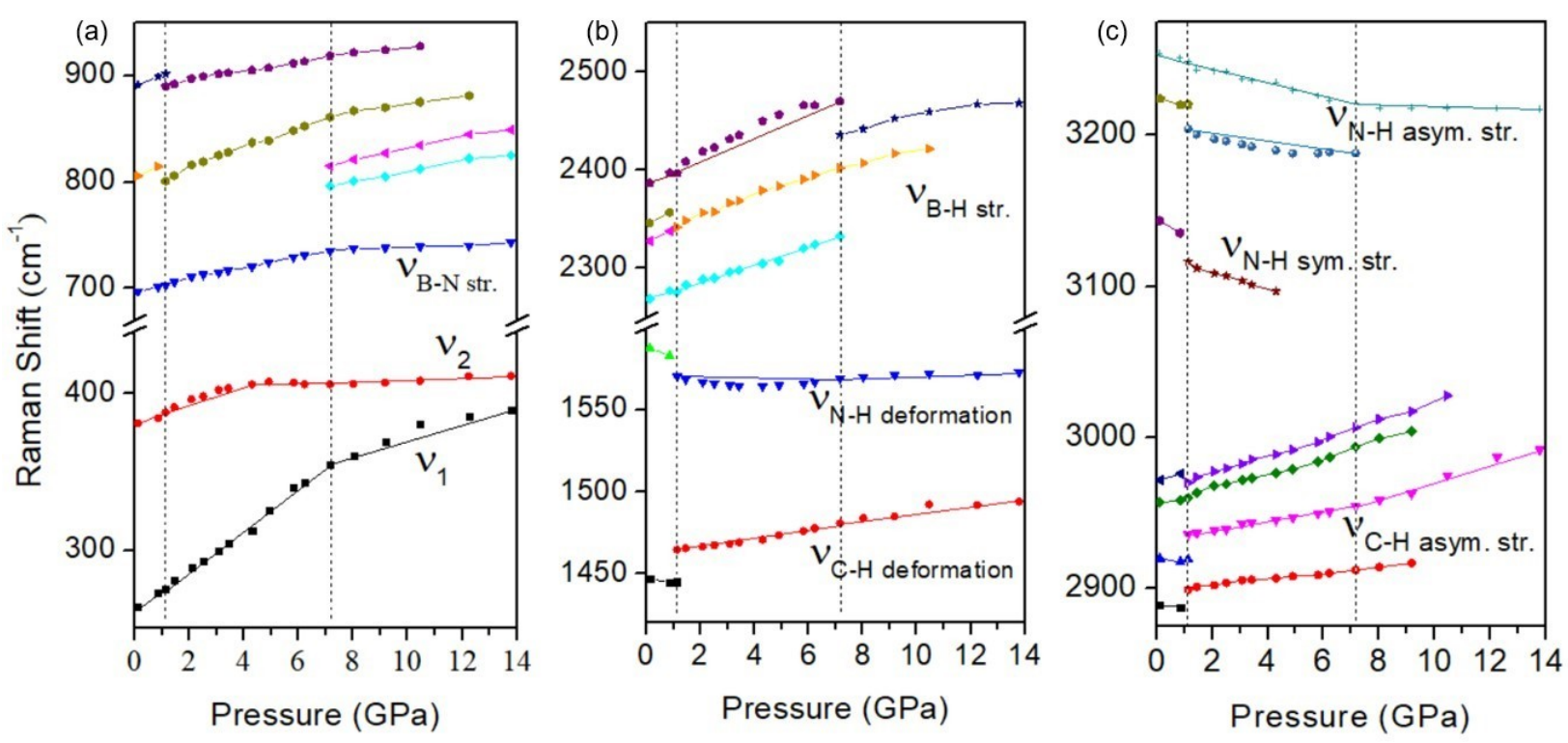

Figure S1. Pressure dependence of the observed vibrational Raman modes of EDAB.

Table S4. Calculated vdW-DF lattice parameters of the 14 candidate structures for phase II at $1.72 \mathrm{GPa}$

\begin{tabular}{rrrrrrrr}
\hline System & $a(\AA)$ & \multicolumn{1}{c}{$b(\AA)$} & \multicolumn{1}{c}{$c(\AA)$} & $\alpha\left(^{\circ}\right)$ & $\beta\left({ }^{\circ}\right)$ & $\gamma\left({ }^{\circ}\right)$ & $V\left(\AA^{3}\right)$ \\
\hline Monoclinic & 10.781 & 9.355 & 7.102 & 90.0 & 115.3 & 90.0 & 647 \\
\hline Monoclinic & 10.060 & 9.355 & 7.102 & 90.0 & 104.4 & 90.0 & 647 \\
\hline Monoclinic & 10.247 & 7.031 & 9.327 & 90.0 & 108.7 & 90.0 & 637 \\
\hline Monoclinic & 8.325 & 10.482 & 8.092 & 90.0 & 103.2 & 90.0 & 687 \\
\hline Monoclinic & 8.143 & 10.941 & 7.433 & 90.0 & 95.2 & 90.0 & 659 \\
\hline Monoclinic & 8.152 & 11.015 & 7.330 & 90.0 & 95.5 & 90.0 & 655 \\
\hline Monoclinic & 11.226 & 6.383 & 10.419 & 90.0 & 106.6 & 90.0 & 715 \\
\hline Monoclinic & 11.365 & 6.445 & 10.628 & 90.0 & 106.6 & 90.0 & 722 \\
\hline Monoclinic & 8.140 & 10.464 & 6.904 & 90.0 & 85.0 & 90.0 & 586 \\
\hline Monoclinic & 11.446 & 8.103 & 6.077 & 90.0 & 86.7 & 90.0 & 563 \\
\hline Monoclinic & 8.835 & 10.456 & 6.537 & 90.0 & 84.6 & 90.0 & 601 \\
\hline Monoclinic & 6.781 & 11.198 & 9.219 & 90.0 & 116.8 & 90.0 & 625 \\
\hline Monoclinic & 10.847 & 11.090 & 7.973 & 90.0 & 103.1 & 90.0 & 583 \\
\hline Monoclinic & 8.152 & 11.015 & 7.330 & 90.0 & 95.5 & 90.0 & 655 \\
\hline
\end{tabular}


Table S5. Calculated vdW-DF lattice parameters of the 17 candidate structures for phase III at 11.44 GPa.

\begin{tabular}{lrrrrrrr}
\hline \multicolumn{1}{c}{ System } & $a(\AA)$ & $b(\AA)$ & \multicolumn{1}{c}{$c(\AA)$} & $\alpha\left(^{\circ}\right)$ & $\beta\left(^{\circ}\right)$ & $\gamma\left({ }^{\circ}\right)$ & $V\left(\AA^{3}\right)$ \\
\hline Orthorhombic & 11.785 & 5.400 & 7.832 & 90.0 & 90.0 & 90.0 & 498 \\
\hline Monoclinic & 7.597 & 7.952 & 8.241 & 90.0 & 104.6 & 90.0 & 482 \\
\hline Monoclinic & 8.251 & 11.303 & 5.374 & 90.0 & 98.8 & 90.0 & 495 \\
\hline Orthorhombic & 12.358 & 3.803 & 10.171 & 90.0 & 90.0 & 90.0 & 475 \\
\hline Orthorhombic & 11.704 & 10.562 & 4.074 & 90.0 & 90.0 & 90.0 & 504 \\
\hline Monoclinic & 8.139 & 10.597 & 5.827 & 90.0 & 88.2 & 90.0 & 502 \\
\hline Monoclinic & 8.755 & 6.314 & 10.413 & 90.0 & 124.8 & 90.0 & 473 \\
\hline Monoclinic & 11.041 & 3.859 & 10.686 & 90.0 & 86.4 & 90.0 & 454 \\
\hline Monoclinic & 10.334 & 7.721 & 5.354 & 90.0 & 85.4 & 90.0 & 426 \\
\hline Monoclinic & 9.625 & 4.369 & 10.831 & 90.0 & 108.2 & 90.0 & 433 \\
\hline Monoclinic & 7.239 & 10.687 & 5.561 & 90.0 & 87.6 & 90.0 & 430 \\
\hline Monoclinic & 7.737 & 8.176 & 6.873 & 90.0 & 85.4 & 90.0 & 433 \\
\hline Monoclinic & 6.926 & 12.475 & 5.762 & 90.0 & 121.0 & 90.0 & 428 \\
\hline Monoclinic & 7.253 & 9.879 & 7.089 & 90.0 & 118.0 & 90.0 & 447 \\
\hline Monoclinic & 3.857 & 10.661 & 7.151 & 90.0 & 128.3 & 90.0 & 460 \\
\hline Orthorhombic & 3.812 & 11.609 & 10.880 & 90.0 & 90.0 & 90.0 & 482 \\
\hline Orthorhombic & 6.176 & 5.538 & 14.178 & 90.0 & 90.0 & 90.0 & 485 \\
\hline
\end{tabular}


Table S6. Calculated vdW-DF enthalpies (eV/molecule) of the final 7 candidate EDAB structures relative to the enthalpy of the reference Pbca structure at the corresponding pressure.

\begin{tabular}{|c|c|c|c|c|c|c|c|}
\hline $\begin{array}{c}P \\
(\mathrm{GPa})\end{array}$ & $C 2 / c$ & $C c$ & $P 2_{1}$ & $P 2_{1} / c(\mathrm{~A})$ & $P 2_{1} / c(\mathrm{~B})$ & $P 2_{1} 2_{1} 2_{1}(\mathrm{~A})$ & $P 212121$ (B) \\
\hline 0 & 0.287 & 0.212 & 0.319 & 0.012 & 0.223 & 0.393 & 0.338 \\
\hline 1 & 0.190 & 0.107 & 0.172 & -0.095 & 0.058 & 0.264 & 0.192 \\
\hline 2 & 0.107 & 0.012 & 0.048 & -0.192 & -0.080 & 0.156 & 0.068 \\
\hline 3 & 0.034 & -0.072 & -0.055 & -0.281 & -0.198 & 0.068 & -0.039 \\
\hline 4 & -0.016 & -0.132 & -0.129 & -0.349 & -0.288 & 0.007 & -0.118 \\
\hline 5 & -0.053 & -0.176 & -0.184 & -0.403 & -0.359 & -0.034 & -0.180 \\
\hline 6 & -0.087 & -0.215 & -0.232 & -0.453 & -0.423 & -0.070 & -0.236 \\
\hline 7 & -0.118 & -0.251 & -0.274 & -0.500 & -0.481 & -0.103 & -0.288 \\
\hline 8 & -0.147 & -0.283 & -0.312 & -0.544 & -0.535 & -0.132 & -0.336 \\
\hline 9 & -0.174 & -0.313 & -0.348 & -0.586 & -0.585 & -0.159 & -0.382 \\
\hline 10 & -0.200 & -0.341 & -0.381 & -0.626 & -0.633 & -0.186 & -0.426 \\
\hline 11 & -0.225 & -0.367 & -0.411 & -0.664 & -0.677 & -0.210 & -0.468 \\
\hline 12 & -0.248 & -0.391 & -0.438 & -0.700 & -0.720 & -0.233 & -0.507 \\
\hline 13 & -0.299 & -0.414 & -0.465 & -0.735 & -0.761 & -0.255 & -0.545 \\
\hline 14 & -0.325 & -0.435 & -0.490 & -0.769 & -0.800 & -0.276 & -0.581 \\
\hline 15 & -0.349 & -0.457 & -0.514 & -0.802 & -0.837 & -0.296 & -0.616 \\
\hline
\end{tabular}




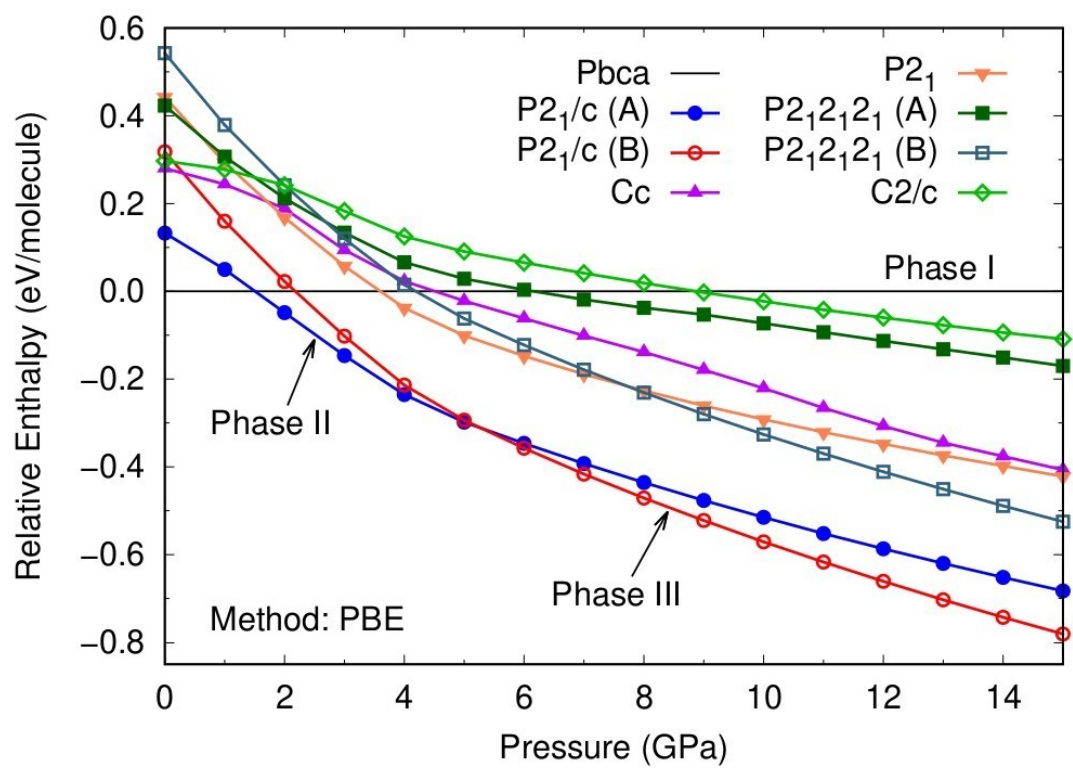

Figure S2. Calculated PBE enthalpies of the final 7 candidate EDAB structures relative to the enthalpy of the reference Pbca structure at the corresponding pressure.
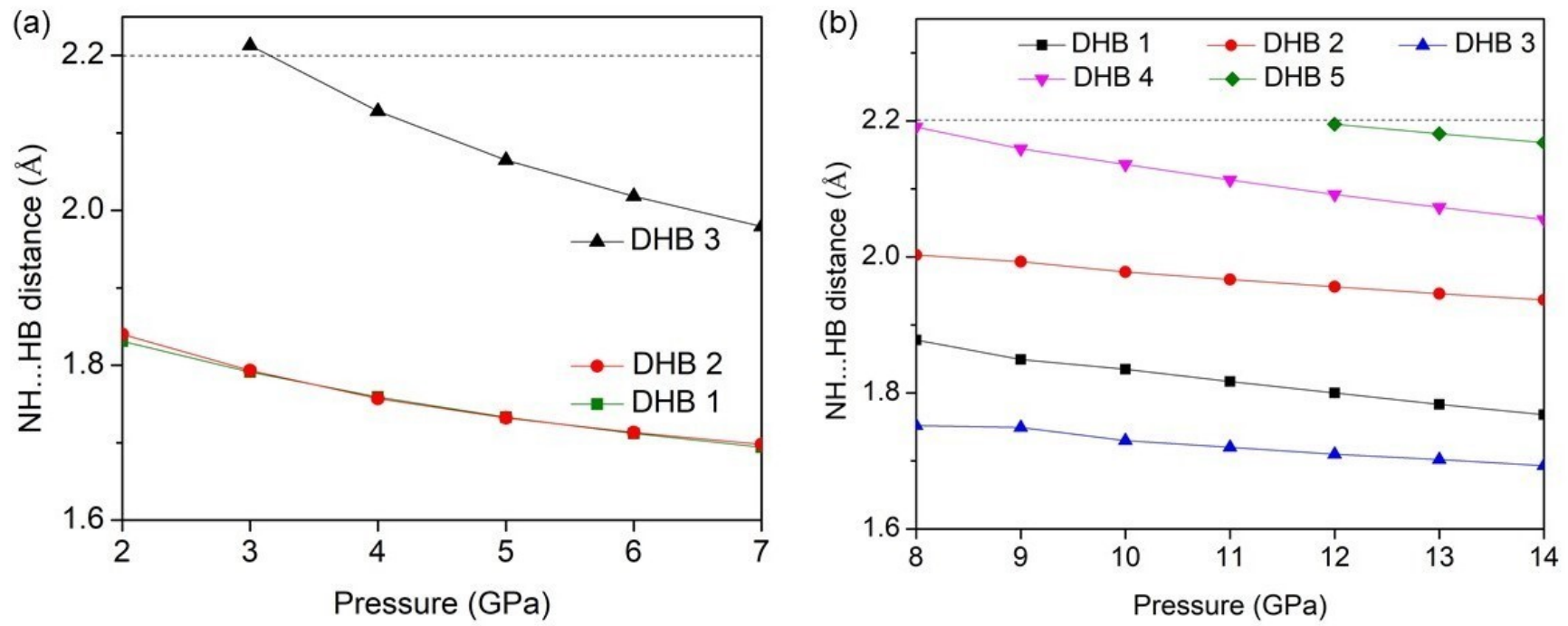

Figure S3. Calculated (vdW-DF) $\mathrm{N}-\mathrm{H} \cdots \mathrm{H}-\mathrm{B}$ dihydrogen bond (DHB) lengths in EDAB vs. pressure: (a) in phase II; (b) in phase III. Different DHB types are distinguished by the $\mathrm{H} \cdots \mathrm{H}$ distance. 
Table S7. Crystal structures, cell parameters ( $a, b, c$ in $\AA ; \alpha, \beta, \gamma$ in degrees) and Wyckoff positions for the unit cells of the 7 candidate structures of EDAB optimized at ambient pressure using the vdW-DF functional.

$C 2 / c$

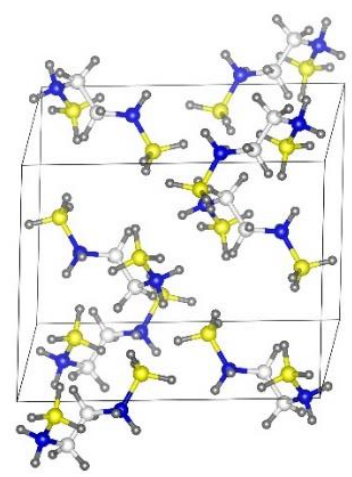

\begin{tabular}{|c|c|c|c|}
\hline$a, b, c$ & 10.723 & 11.879 & 14.265 \\
\hline$\alpha, \beta, \gamma$ & 90.000 & 132.643 & 90.000 \\
\hline $\mathrm{N}(8 f)$ & 0.5737 & 0.1620 & 0.6022 \\
\hline $\mathrm{N}(8 f)$ & 0.1839 & -0.0832 & 0.5749 \\
\hline$C(8 f)$ & 0.6061 & 0.2752 & 0.6638 \\
\hline$C(8 f)$ & 0.2466 & -0.1585 & 0.6855 \\
\hline $\mathrm{B}(8 f)$ & 0.4052 & 0.0947 & 0.5547 \\
\hline $\mathrm{B}(8 f)$ & 0.5390 & 0.3694 & 0.4293 \\
\hline $\mathrm{H}(8 f)$ & 0.4353 & -0.0057 & 0.5612 \\
\hline $\mathrm{H}(8 f)$ & 0.2867 & 0.1 & 0.4459 \\
\hline $\mathrm{H}(8 f)$ & 0.3862 & 0.1201 & 0.6264 \\
\hline $\mathrm{H}(8 f)$ & 0.5627 & 0.1730 & 0.5255 \\
\hline $\mathrm{H}(8 f)$ & 0.6806 & 0.1139 & 0.6655 \\
\hline $\mathrm{H}(8 f)$ & 0.6441 & 0.2584 & 0.7554 \\
\hline $\mathrm{H}(8 f)$ & 0.4860 & 0.3211 & 0.6043 \\
\hline $\mathrm{H}(8 f)$ & 0.3398 & -0.2162 & 0.7024 \\
\hline $\mathrm{H}(8 f)$ & 0.3127 & -0.1052 & 0.7700 \\
\hline $\mathrm{H}(8 f)$ & 0.2871 & -0.0594 & 0.5880 \\
\hline $\mathrm{H}(8 f)$ & 0.1435 & -0.0090 & 0.5840 \\
\hline $\mathrm{H}(8 f)$ & 0.5909 & 0.2817 & 0.4228 \\
\hline $\mathrm{H}(8 f)$ & 0.4101 & 0.3542 & 0.4088 \\
\hline $\mathrm{H}(8 f)$ & 0.5201 & 0.4412 & 0.3600 \\
\hline
\end{tabular}

$C c$

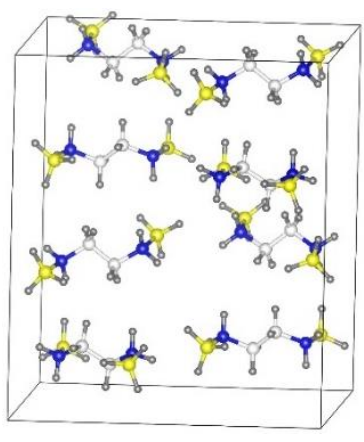

\begin{tabular}{|r|r|r|r|}
\hline \hline$a, b, c$ & 16.999 & 15.149 & 5.441 \\
\hline$\alpha, \beta, \gamma$ & 90.000 & 108.341 & 90.000 \\
\hline $\mathrm{N}(4 a)$ & 0.8496 & 0.1320 & 0.7812 \\
\hline $\mathrm{N}(4 a)$ & 0.3225 & -0.1197 & 0.6296 \\
\hline $\mathrm{N}(4 a)$ & 0.6040 & -0.1205 & 0.4146 \\
\hline $\mathrm{N}(4 a)$ & 0.5746 & -0.3689 & 0.5053 \\
\hline $\mathrm{C}(4 a)$ & 0.3091 & -0.2829 & 0.6591 \\
\hline $\mathrm{C}(4 a)$ & 0.3659 & -0.2031 & 0.7535 \\
\hline $\mathrm{C}(4 a)$ & 0.5601 & -0.2037 & 0.4520 \\
\hline $\mathrm{C}(4 a)$ & 0.6153 & -0.2844 & 0.4605 \\
\hline $\mathrm{B}(4 a)$ & 0.8588 & 0.1148 & 0.0857 \\
\hline $\mathrm{B}(4 a)$ & 0.3210 & -0.0989 & 0.3324 \\
\hline $\mathrm{B}(4 a)$ & 0.6053 & -0.0988 & 0.1207 \\
\hline $\mathrm{B}(4 a)$ & 0.5670 & -0.3855 & 0.7937 \\
\hline $\mathrm{H}(4 a)$ & 0.4000 & -0.3258 & 0.2127 \\
\hline $\mathrm{H}(4 a)$ & 0.8924 & 0.0439 & 0.1401 \\
\hline $\mathrm{H}(4 a)$ & 0.7891 & 0.1149 & 0.1036 \\
\hline $\mathrm{H}(4 a)$ & 0.8159 & 0.0806 & 0.6763 \\
\hline $\mathrm{H}(4 a)$ & 0.4072 & -0.3732 & 0.7571 \\
\hline $\mathrm{H}(4 a)$ & 0.7521 & 0.2240 & 0.7102 \\
\hline $\mathrm{H}(4 a)$ & 0.7931 & 0.2106 & 0.4491 \\
\hline $\mathrm{H}(4 a)$ & 0.4225 & -0.2089 & 0.7001 \\
\hline $\mathrm{H}(4 a)$ & 0.3822 & -0.1964 & 0.9632 \\
\hline $\mathrm{H}(4 a)$ & 0.3495 & -0.0667 & 0.7428 \\
\hline $\mathrm{H}(4 a)$ & 0.2626 & -0.1202 & 0.6353 \\
\hline $\mathrm{H}(4 a)$ & 0.3896 & -0.0708 & 0.3464 \\
\hline $\mathrm{H}(4 a)$ & 0.3056 & -0.1672 & 0.2118 \\
\hline $\mathrm{H}(4 a)$ & 0.2664 & -0.0449 & 0.2474 \\
\hline $\mathrm{H}(4 a)$ & 0.6597 & -0.0443 & 0.1445 \\
\hline $\mathrm{H}(4 a)$ & 0.5367 & -0.0709 & 1.0004 \\
\hline $\mathrm{H}(4 a)$ & 0.6212 & -0.1666 & 0.0288 \\
\hline $\mathrm{H}(4 a)$ & 0.5774 & -0.0675 & 0.4764 \\
\hline $\mathrm{H}(4 a)$ & 0.6640 & -0.1215 & 0.5375 \\
\hline $\mathrm{H}(4 a)$ & 0.5025 & -0.2080 & 0.2904 \\
\hline $\mathrm{H}(4 a)$ & 0.5456 & -0.1978 & 0.6328 \\
\hline $\mathrm{H}(4 a)$ & 0.6736 & -0.2786 & 0.6193 \\
\hline $\mathrm{H}(4 a)$ & 0.6290 & -0.2906 & 0.2781 \\
\hline $\mathrm{H}(4 a)$ & 0.1075 & 0.0793 & 0.4646 \\
\hline $\mathrm{H}(4 a)$ & 0.5166 & -0.3737 & 0.3700 \\
\hline $\mathrm{H}(4 a)$ & 0.1370 & 0.1151 & 0.9461 \\
\hline $\mathrm{H}(4 a)$ & 0.5258 & -0.3262 & 0.8403 \\
\hline $\mathrm{H}(4 a)$ & 0.5337 & -0.4565 & 0.7849 \\
\hline & & & \\
\hline
\end{tabular}

$P 2_{1}$

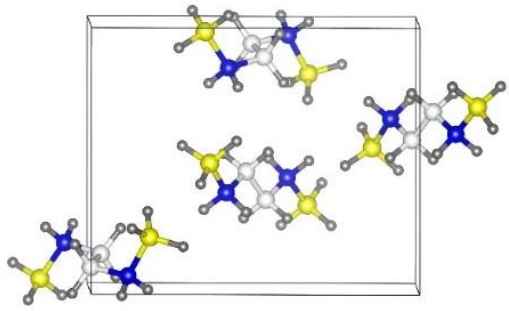

\begin{tabular}{|r|r|r|r|}
\hline \hline$a, b, c$ & 9.117 & 11.183 & 6.254 \\
\hline$\alpha, \beta, \gamma$ & 90.000 & 97.799 & 90.000 \\
\hline $\mathrm{N}(2 a)$ & 0.3608 & 0.5829 & 0.5061 \\
\hline $\mathrm{N}(2 a)$ & 0.3962 & 0.3905 & 1.0068 \\
\hline $\mathrm{N}(2 a)$ & 0.1727 & 0.0757 & 0.5255 \\
\hline $\mathrm{N}(2 a)$ & 0.0705 & 0.8884 & -0.0021 \\
\hline $\mathrm{C}(2 a)$ & 0.4328 & 0.5200 & 0.3352 \\
\hline $\mathrm{C}(2 a)$ & 0.3200 & 0.4582 & 0.1671 \\
\hline $\mathrm{C}(2 a)$ & 0.1375 & 0.9665 & 0.6509 \\
\hline $\mathrm{C}(2 a)$ & 0.0963 & 0.0005 & 0.8729 \\
\hline $\mathrm{B}(2 a)$ & 0.4792 & 0.6361 & 0.7037 \\
\hline $\mathrm{B}(2 a)$ & 0.2848 & 0.3270 & 0.8150 \\
\hline $\mathrm{B}(2 a)$ & 0.0322 & 0.1491 & 0.3968 \\
\hline $\mathrm{B}(2 a)$ & 0.2208 & 0.8247 & 0.1213 \\
\hline $\mathrm{H}(2 a)$ & 0.4083 & 0.6652 & 0.8447 \\
\hline $\mathrm{H}(2 a)$ & 0.5426 & 0.7189 & 0.6315 \\
\hline $\mathrm{H}(2 a)$ & 0.5650 & 0.5553 & 0.7610 \\
\hline $\mathrm{H}(2 a)$ & 0.2940 & 0.6502 & 0.4357 \\
\hline $\mathrm{H}(2 a)$ & 0.3112 & 0.8285 & 0.9984 \\
\hline $\mathrm{H}(2 a)$ & 0.5089 & 0.4538 & 0.4182 \\
\hline $\mathrm{H}(2 a)$ & 0.4960 & 0.5871 & 0.2585 \\
\hline $\mathrm{H}(2 a)$ & 0.2472 & 0.5240 & 0.0770 \\
\hline $\mathrm{H}(2 a)$ & 0.2538 & 0.3936 & 0.2446 \\
\hline $\mathrm{H}(2 a)$ & 0.4626 & 0.3257 & 0.0866 \\
\hline $\mathrm{H}(2 a)$ & 0.4662 & 0.4474 & 0.9398 \\
\hline $\mathrm{H}(2 a)$ & 0.2117 & 0.2554 & 0.9001 \\
\hline $\mathrm{H}(2 a)$ & 0.2064 & 0.4049 & 0.7243 \\
\hline $\mathrm{H}(2 a)$ & 0.3625 & 0.2813 & 0.6952 \\
\hline $\mathrm{H}(2 a)$ & 0.0885 & 0.2298 & 0.3056 \\
\hline $\mathrm{H}(2 a)$ & 0.9581 & 0.1864 & 0.5282 \\
\hline $\mathrm{H}(2 a)$ & 0.9652 & 0.0799 & 0.2701 \\
\hline $\mathrm{H}(2 a)$ & 0.2355 & 0.1347 & 0.6259 \\
\hline $\mathrm{H}(2 a)$ & 0.2394 & 0.0489 & 0.4145 \\
\hline $\mathrm{H}(2 a)$ & 0.2336 & 0.9068 & 0.6703 \\
\hline $\mathrm{H}(2 a)$ & 1.0463 & 0.9195 & 0.5541 \\
\hline $\mathrm{H}(2 a)$ & 0.9972 & 0.0565 & 0.8564 \\
\hline $\mathrm{H}(2 a)$ & 0.1860 & 0.0502 & 0.9677 \\
\hline $\mathrm{H}(2 a)$ & 1.0138 & 0.8270 & 0.8952 \\
\hline $\mathrm{H}(2 a)$ & 1.0008 & 0.9087 & 0.1085 \\
\hline $\mathrm{H}(2 a)$ & 0.2914 & 0.5239 & 0.5694 \\
\hline $\mathrm{H}(2 a)$ & 0.2596 & 0.8805 & 0.2851 \\
\hline $\mathrm{H}(2 a)$ & 0.1867 & 0.7227 & 0.1581 \\
\hline & & & \\
\hline
\end{tabular}


$P 2{ }_{1} / c$ (A)

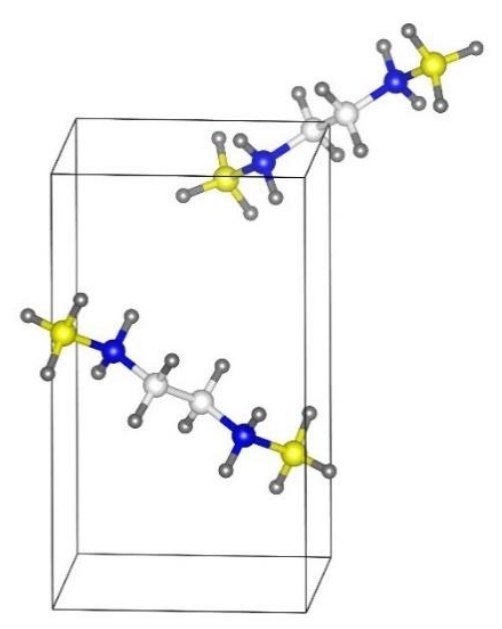

\begin{tabular}{|l|r|r|r|}
\hline \hline$a, b, c$ & 4.744 & 6.705 & 10.728 \\
\hline$\alpha, \beta, \gamma$ & 90.000 & 103.220 & 90.000 \\
\hline $\mathrm{N}(4 e)$ & 0.0175 & 0.2509 & 1.4125 \\
\hline $\mathrm{C}(4 e)$ & 0.8850 & 0.4260 & 1.4654 \\
\hline $\mathrm{B}(4 e)$ & 0.7794 & 0.0892 & 1.3379 \\
\hline $\mathrm{H}(4 e)$ & 0.9177 & 0.9471 & 1.3139 \\
\hline $\mathrm{H}(4 e)$ & 0.6411 & 0.1709 & 1.2426 \\
\hline $\mathrm{H}(4 e)$ & 0.6279 & 0.0447 & 1.4108 \\
\hline $\mathrm{H}(4 e)$ & 0.1351 & 0.3021 & 1.3495 \\
\hline $\mathrm{H}(4 e)$ & 0.1600 & 0.1792 & 1.4855 \\
\hline $\mathrm{H}(4 e)$ & 0.7611 & 0.3658 & 0.5314 \\
\hline $\mathrm{H}(4 e)$ & 0.7357 & 0.4975 & 1.3847 \\
\hline
\end{tabular}

$P 2{ }_{1} / c$ (B)

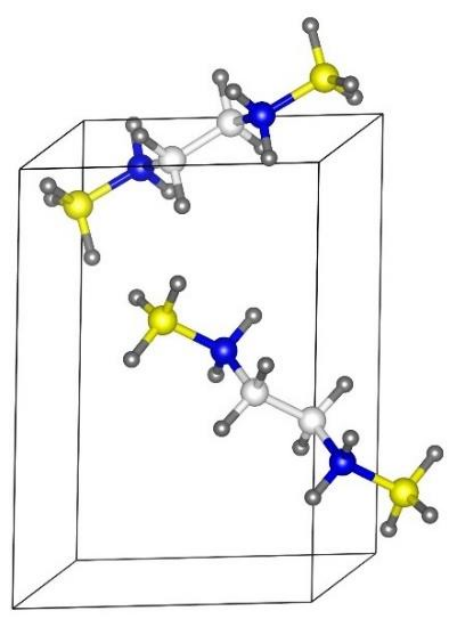

\begin{tabular}{|l|r|r|r|}
\hline \hline$a, b, c$ & 4.489 & 11.129 & 7.162 \\
\hline$\alpha, \beta, \gamma$ & 90.000 & 119.982 & 90.000 \\
\hline $\mathrm{N}(4 e)$ & -0.7009 & -0.9037 & 0.2474 \\
\hline $\mathrm{C}(4 e)$ & -1.0237 & -0.9674 & 0.0862 \\
\hline $\mathrm{B}(4 e)$ & 0.2752 & -0.8390 & 0.4445 \\
\hline $\mathrm{H}(4 e)$ & 0.5588 & -0.7993 & 0.5656 \\
\hline $\mathrm{H}(4 e)$ & 0.1920 & -0.9160 & 0.5299 \\
\hline $\mathrm{H}(4 e)$ & 0.0564 & -0.7622 & 0.3660 \\
\hline $\mathrm{H}(4 e)$ & -0.5017 & -0.9647 & 0.3132 \\
\hline $\mathrm{H}(4 e)$ & -0.6382 & -0.8399 & 0.1686 \\
\hline $\mathrm{H}(4 e)$ & 0.7742 & -0.8991 & 1.0124 \\
\hline $\mathrm{H}(4 e)$ & -1.0886 & -0.0314 & 0.1764 \\
\hline
\end{tabular}

$P 2{ }_{1} 2_{1}$ (A)

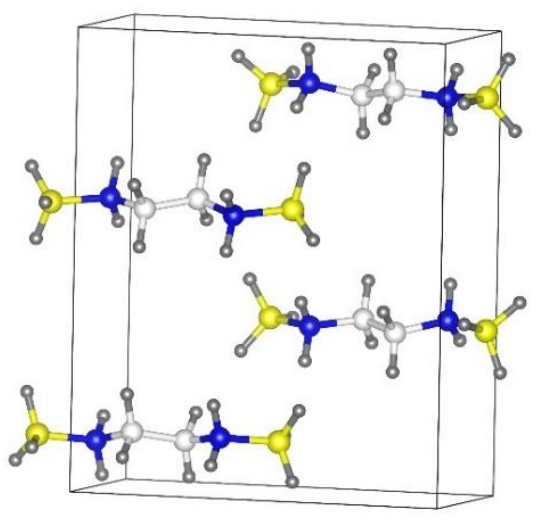

\begin{tabular}{|r|r|r|r|}
\hline \hline$a, b, c$ & 4.754 & 12.741 & 10.589 \\
\hline$\alpha, \beta, \gamma$ & 90.000 & 90.000 & 90.000 \\
\hline $\mathrm{N}(4 a)$ & 0.5867 & 0.1339 & 0.0816 \\
\hline $\mathrm{N}(4 a)$ & 1.4599 & 0.1122 & 0.4380 \\
\hline $\mathrm{C}(4 a)$ & 1.4223 & 0.1192 & 0.2163 \\
\hline $\mathrm{C}(4 a)$ & 0.6099 & 0.1389 & 0.3174 \\
\hline $\mathrm{B}(4 a)$ & 0.4137 & 0.1251 & 0.9473 \\
\hline $\mathrm{B}(4 a)$ & 0.6403 & 0.1249 & 0.5698 \\
\hline $\mathrm{H}(4 a)$ & 0.4935 & 0.1967 & 0.8813 \\
\hline $\mathrm{H}(4 a)$ & 1.1630 & 0.1311 & 0.9705 \\
\hline $\mathrm{H}(4 a)$ & 0.4745 & 0.0424 & 0.8994 \\
\hline $\mathrm{H}(4 a)$ & 0.6854 & 0.2058 & 0.0850 \\
\hline $\mathrm{H}(4 a)$ & 0.7442 & 0.0786 & 0.0808 \\
\hline $\mathrm{H}(4 a)$ & 1.3399 & 0.0389 & 0.2012 \\
\hline $\mathrm{H}(4 a)$ & 1.2465 & 0.1745 & 0.1993 \\
\hline $\mathrm{H}(4 a)$ & 0.6751 & 0.2212 & 0.3227 \\
\hline $\mathrm{H}(4 a)$ & 0.7976 & 0.0893 & 0.3136 \\
\hline $\mathrm{H}(4 a)$ & 1.3833 & 0.0370 & 0.4314 \\
\hline $\mathrm{H}(4 a)$ & 1.2849 & 0.1595 & 0.4449 \\
\hline $\mathrm{H}(4 a)$ & 0.8842 & 0.1049 & 0.5475 \\
\hline $\mathrm{H}(4 a)$ & 0.6089 & 0.2146 & 0.6051 \\
\hline $\mathrm{H}(4 a)$ & 0.5361 & 0.0641 & 0.6444 \\
\hline
\end{tabular}


$P 2{ }_{1} 2_{1}{ }_{1}$ (B)

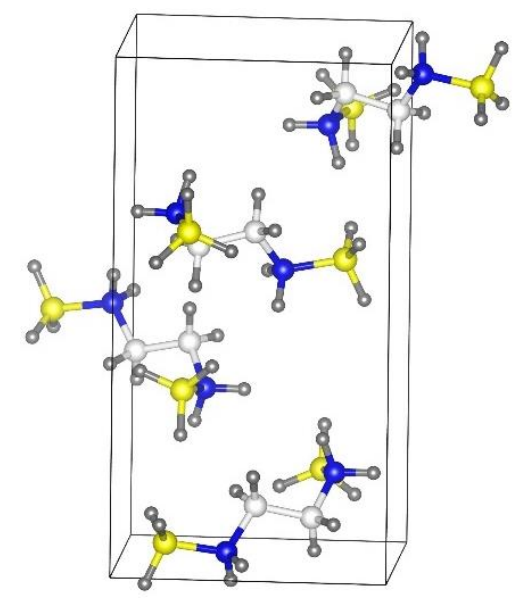

\begin{tabular}{|r|r|r|r|}
\hline \hline$a, b, c$ & 13.889 & 6.274 & 7.259 \\
\hline$\alpha, \beta, \gamma$ & 90.000 & 90.000 & 90.000 \\
\hline $\mathrm{N}(4 a)$ & 0.6874 & 0.8142 & 0.2138 \\
\hline $\mathrm{N}(4 a)$ & 0.5568 & 0.4802 & 0.5771 \\
\hline $\mathrm{C}(4 a)$ & 0.6130 & 0.6582 & 0.2835 \\
\hline $\mathrm{C}(4 a)$ & 0.6378 & 0.5957 & 0.4822 \\
\hline $\mathrm{B}(4 a)$ & 0.6726 & 0.0635 & 0.2776 \\
\hline $\mathrm{B}(4 a)$ & 0.5758 & 0.4319 & 0.7963 \\
\hline $\mathrm{H}(4 a)$ & 0.7516 & 0.1449 & 0.2760 \\
\hline $\mathrm{H}(4 a)$ & 0.6379 & 0.0634 & 0.4308 \\
\hline $\mathrm{H}(4 a)$ & 0.6191 & 0.1434 & 0.1648 \\
\hline $\mathrm{H}(4 a)$ & 0.7548 & 0.7635 & 0.2541 \\
\hline $\mathrm{H}(4 a)$ & 0.6888 & 0.8087 & 0.0723 \\
\hline $\mathrm{H}(4 a)$ & 0.6114 & 0.5185 & 0.1933 \\
\hline $\mathrm{H}(4 a)$ & 0.5429 & 0.7376 & 0.2780 \\
\hline $\mathrm{H}(4 a)$ & 0.6531 & 0.7371 & 0.5645 \\
\hline $\mathrm{H}(4 a)$ & 0.7011 & 0.4919 & 0.4847 \\
\hline $\mathrm{H}(4 a)$ & 0.5435 & 0.3385 & 0.5110 \\
\hline $\mathrm{H}(4 a)$ & 0.4958 & 0.5721 & 0.5670 \\
\hline $\mathrm{H}(4 a)$ & 0.6228 & 0.2686 & 0.8055 \\
\hline $\mathrm{H}(4 a)$ & 0.6185 & 0.5841 & 0.8587 \\
\hline $\mathrm{H}(4 a)$ & 0.4976 & 0.4101 & 0.8665 \\
\hline
\end{tabular}

Pbca

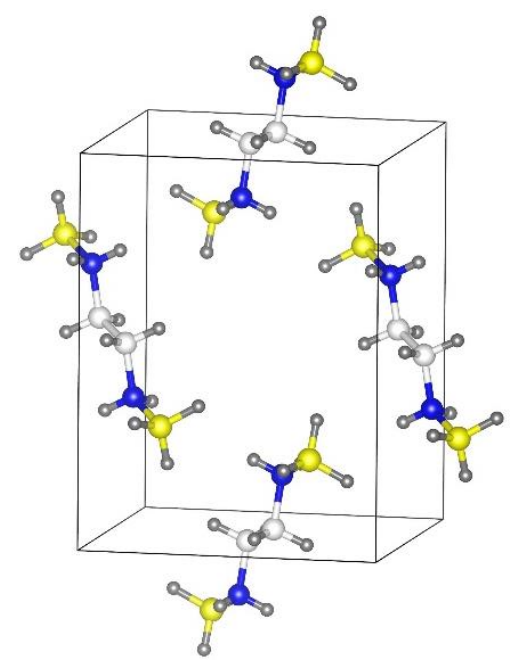

\begin{tabular}{|r|r|r|r|}
\hline \hline$a, b, c$ & 10.669 & 8.345 & 8.236 \\
\hline$\alpha, \beta, \gamma$ & 90.000 & 90.000 & 90.000 \\
\hline $\mathrm{N}(8 c)$ & 0.6631 & 0.0847 & 1.0501 \\
\hline $\mathrm{C}(8 c)$ & 0.5245 & 0.0564 & 1.0663 \\
\hline $\mathrm{B}(8 c)$ & 0.7214 & 0.2030 & 1.1870 \\
\hline $\mathrm{H}(8 c)$ & 0.6806 & 0.1312 & 0.9368 \\
\hline $\mathrm{H}(8 c)$ & 0.7089 & 0.9765 & 1.0557 \\
\hline $\mathrm{H}(8 c)$ & 0.5082 & 0.0066 & 1.1875 \\
\hline $\mathrm{H}(8 c)$ & 0.4785 & 0.1735 & 1.0600 \\
\hline $\mathrm{H}(8 c)$ & 0.6658 & 0.3301 & 1.1749 \\
\hline $\mathrm{H}(8 c)$ & 0.7021 & 0.1404 & 1.3180 \\
\hline $\mathrm{H}(8 c)$ & 0.8328 & 0.2161 & 1.1590 \\
\hline
\end{tabular}

